BMJ Open Sport \& Exercise Medicine

\title{
Return to play following anterior cruciate ligament reconstruction: incorporating fatigue into a return to play functional battery. Part A: treadmill running
}

\author{
Richard Connell, ${ }^{\circledR}$ Ross Milne, Bruce Paton ${ }^{\odot}$
}

To cite: Connell R, Milne R, Paton B. Return to play following anterior cruciate ligament reconstruction: incorporating fatigue into a return to play functional battery. Part A: treadmill running. BMJ Open Sport \& Exercise Medicine 2019;5:e000375. doi:10.1136/ bmjsem-2018-000375

- Additional material is published online only. To view please visit the journal online (http://dx.doi.org/10.1136/ bmjsem-2018-000375)

Accepted 31 January 2019
Check for updates

C Author(s) (or their employer(s)) 2019. Re-use permitted under CC BY-NC. No commercial re-use. See rights and permissions. Published by BMJ.

The Institute of Sport, Exercise \& Health, London, UK

Correspondence to Dr Richard Connell; rjrconnell@doctors.org.uk

\section{ABSTRACT}

Background The risk of reinjury and other sequelae following anterior cruciate ligament reconstruction (ACLR) remains high. Lack of knowledge regarding factors contributing to these risks limits our ability to develop sensitive return to play (RTP) tests. Using a running task, we evaluate whether fatigue induces alterations in foot progression angle (FPA), a proposed biomechanical risk factor and could be used to enhance RTP test sensitivity. Method Transverse plane foot kinematics (FPA) were assessed for 18 post-ACLR subjects during a treadmill running task, before and after a generalised lower limb fatigue protocol. Subject's contralateral limbs were used as a control group.

Results A small but significant difference between FPA for ACLR and contralateral limbs was observed before but not after fatigue. When confounding variables were considered, there was a significant difference in FPA change between ACLR and contralateral limbs from the prefatigue to postfatigue state.

Conclusions Following ACLR athletes may develop a knee-protective movement strategy that delays the progression of osteoarthritis in the ACL-injured knee. This may, however, increase the risk of ACL reinjury. Following the onset of fatigue this proposed movement strategy, and thus osteoarthritis protection, is lost.

\section{INTRODUCTION}

Anterior cruciate ligament (ACL) rupture has significant short-term and long-term consequences. Many athletes never return to the same level of performance while rates of reinjury and osteoarthritis remain high. Optimal management including surgical techniques, rehabilitation protocols and return to play (RTP) criteria have not been fully established, contributing to these issues. ACL injury is a strong predictor of subsequent injury; reinjury rates of up to $25 \%$ have been reported. ${ }^{12}$ Risk factors consequent to ACL rupture, therefore, increase rates of graft rupture. ${ }^{3}$ Without incorporating such factors,
What are the new findings?

Following anterior cruciate ligament reconstruction (ACLR), athletes develop a unique, asymmetrical gait. While running, athletes were demonstrated to have externally rotated operated limb feet and internally rotated contralateral limb feet.

- Fatigue abolishes differences in foot symmetry in post-ACLR athletes.

- This may represent a joint-protective movement strategy by reducing knee valgus angulation during each stride and thus delaying the progression of medial knee compartment osteoarthritis in ACLreconstructed knees.

What are the implications of these findings on clinical practice?

Exercising under the influence of fatigue may accelerate the progression of osteoarthritis in anterior cruciate ligament (ACL)-reconstructed knees.

- The risk of ACL rerupture may be greatest prior to the onset of fatigue.

- During rehabilitation, consideration should be given to how fatigue influences lower limb biomechanics in the ACL-reconstructed athlete. This could be used to determine progression towards return to play.

RTP tests will not elicit all relevant functional deficits. ${ }^{4}$ Research suggests that outcomes correlate best with physical performance and several biomechanical variables are predictive of reinjury. ${ }^{15}$ Fatigue is a factor proposed to affect physical performance and therefore reinjury rates.

The risk of sequelae is highest for those returning to sport following ACL reconstruction (ACLR). There is limited awareness of how factors synonymous with sport, such as fatigue, interact with biomechanical variables to alter knee joint loading. ${ }^{6-9}$ Five studies have assessed fatigue's effect on lower limb 
biomechanics post-ACLR, identifying changes in hip and knee biomechanics. ${ }^{10-14}$ If fatigue contributes to the risk of reinjury and other sequelae through alterations in lower limb biomechanics, incorporating it into RTP tests may improve sensitivity. No study has assessed the effect of fatigue on foot progression angles (FPAs) following ACLR despite FPA effecting the risk of ACL rupture and osteoarthritis in healthy populations. FPA causes changes in frontal plane knee biomechanics. An internally rotated FPA (toe in) increases knee valgus moments increasing ACL injury risk. Conversely, an externally rotated FPA (toe out) increases knee adduction moments ${ }^{15}$ which accelerate knee medial compartment osteoarthritis. ${ }^{16}$ Using a treadmill running task, this is the first study is to assess whether fatigue influences FPA post-ACLR. We have chosen an activity and outcome measure that are easily reproducible in clinical practice so could contribute to the development of an enhanced RTP test battery.

\section{METHOD}

\section{Study design}

This is a laboratory-based case-control study with a preintervention/postintervention design. It provides level 3b evidence.

\section{Subjects}

Using a database of ACLR cases conducted at University College London (UCL) hospital between January 2007 and January 2016, we recruited 18 subjects; 14 males, 4 females who underwent ACLR and completed rehabilitation at UCL hospital (figure 1). Subjects eligible for

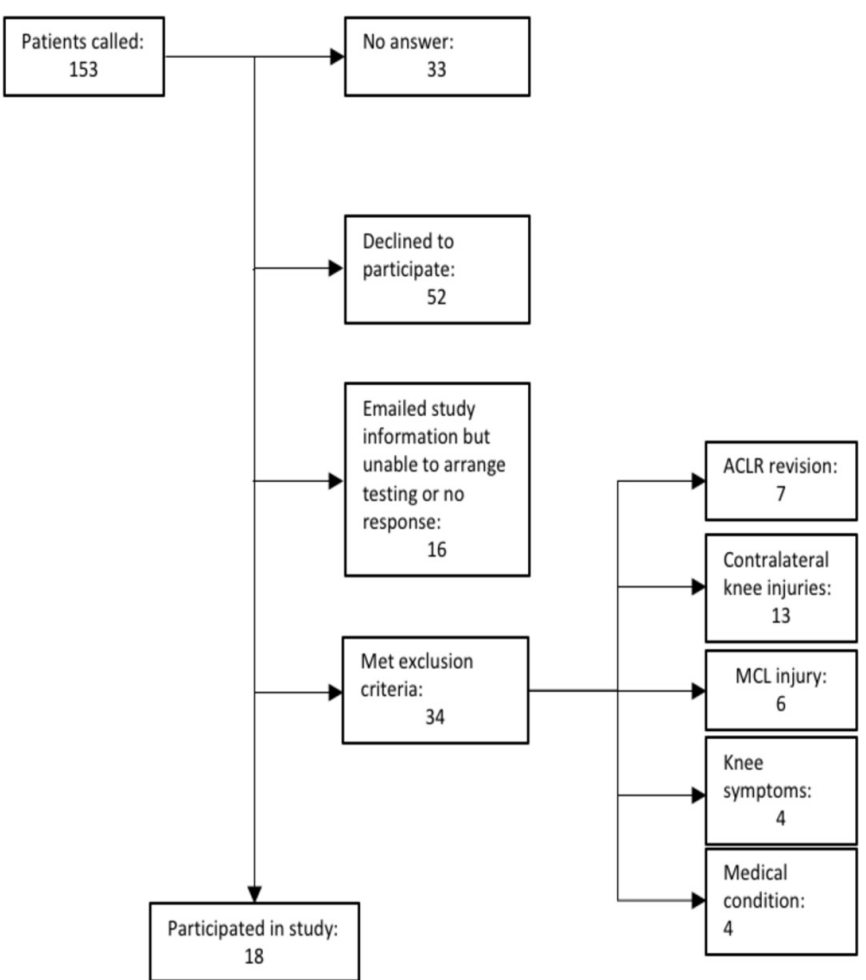

Figure 1 Subject recruitment flow chart (MCL, medial collateral ligament).
Table 1 Procedures performed concurrently with ACLR for study subjects

\begin{tabular}{ll}
\hline Concurrent procedures & $\begin{array}{l}\text { No of } \\
\text { subjects }\end{array}$ \\
\hline No concurrent procedures & 3 \\
\hline Partial medial meniscectomy & 5 \\
\hline Partial lateral meniscectomy & 3 \\
\hline Partial medial and partial lateral meniscectomy & 2 \\
\hline Medial meniscus repair & 1 \\
\hline Chondroplasty & 1 \\
\hline Chondroplasty and partial medial meniscectomy & 2 \\
\hline Chondroplasty and partial lateral meniscectomy & 1 \\
\hline
\end{tabular}

ACLR, anterior cruciate ligament reconstruction.

inclusion were aged between 18 and 65 , had undergone unilateral primary ACLR using hamstring autografts obtained from the same limb and had been discharged from both orthopaedic and physiotherapy care. Subjects were required to be a minimum of 1-year postsurgery and to have returned to recreational activity. In addition, subjects were required to have healthy contralateral knees and to demonstrate proficiency at all the functional tests. Exclusion criteria include:

- Other knee ligaments injured at the same time as the ACL.

- Previous lower limb joint injuries that required consultation with a healthcare professional.

- Medical condition(s) that limit exercise.

- Pain or the sensation of the knee giving way during testing.

Subjects who had undergone partial meniscectomy or chondroplasty were included in the study (table 1). Written informed consent was obtained from all subjects prior to testing.

\section{Pretesting measures}

Each subject's physical activity level was assessed using the Tegner Activity Scale. Both the current level and that preceding injury are reported. The International Knee Documentation Committee (IKDC) subjective knee evaluation, Lysholm score and the Lower Extremity Functional Scale were completed to evaluate knee-related symptoms and function (table 2). Each measure has been demonstrated to be reliable and valid. ${ }^{17-19}$

A clinical examination was conducted on all participants prior to testing. This included measurements of height, weight, body composition and a bilateral knee evaluation. Knee examination comprised an assessment for joint-line tenderness, swelling, laxity (Lachman and anterior drawer tests), range of motion and muscle strength. Subjects were examined by two examiners and a consensus was reached regarding findings. 


\begin{tabular}{ll} 
Table 2 Patient demographics and information \\
\hline Age (years) & $37.7(10.0)$ \\
\hline Gender & 14 male/4 female \\
Weight (kg) & $75.4(10.8)$ \\
IKDC total & $79.2(5.9)$ \\
\hline Lysholm score & $142.0(7.1)$ \\
Current Tegner Activity Score & $5.1(1.9)$ \\
Preinjury Tegner Activity Score & $6.8(1.6)$ \\
LEFS & $76.6(3.55)$ \\
\hline Time since surgery (months) & $45.3(15.4)$ \\
Dominant limb & 7 ACLR, 11 contralateral \\
$\begin{array}{l}\text { Prefatigue running speed (km/ } \\
\text { hour) }\end{array}$ & $12.7(1.8)$ \\
$\begin{array}{l}\text { Postfatigue running speed (km/ } \\
\text { hour) }\end{array}$ & $12.9(1.8)$ \\
\hline
\end{tabular}

Data presented as mean (SD).

ACLR, anterior cruciate ligament reconstruction; IKDC,

International Knee Documentation Committee; LEFS, Lower

Extremity Functional Scale.

\section{Apparatus}

A Kistler $\mathrm{h} / \mathrm{p} /$ cosmos treadmill (Nussdorf-Traunstein, Germany) with built-in force plates was used for testing. Data were recorded using Gaitway software V.2.08 (New York, USA). Participants completed the fatigue protocol on a supine leg press with the weight stack connected to a linear encoder. Ergotest Muscle Lab software (Porsgrunn, Norway) was used to determine power output for each lower limb. Kistler state that their apparatus is reliable and valid, while other studies support this assertion for force plate data. ${ }^{2021}$

\section{Experimental procedure}

Prior to testing, subjects completed a warm-up consisting of jogging for $5 \mathrm{~min}$ at a comfortable pace, 2 sets of 10 squats and 5 drop jumps. Subjects jogged barefoot on the treadmill to acclimatise to running without shoes. Subjects then selected a speed which they could maintain for $1 \mathrm{~min}$ (table 2). Data were gathered for a $15 \mathrm{~s}$ period. Subjects then completed the fatigue protocol described below, before repeating the running task at their previously selected speed. This was performed without delay to ensure that subjects remained maximally fatigued. Each subject completed data collection in a single session.

\section{Fatigue protocol}

The fatigue protocol was a lower limb resistance exercise effective at fatiguing all lower limb muscle groups. ${ }^{22-24}$ While supine on the leg press machine, subjects were instructed to jump maximally with a single leg, land on the same leg and repeat. Unilateral maximal jumps continued until Muscle Lab demonstrated that the power output for that limb had declined by $30 \%$ for two consecutive jumps. Subjects were asked to minimise ground contact time between jumps and were given verbal encouragement to

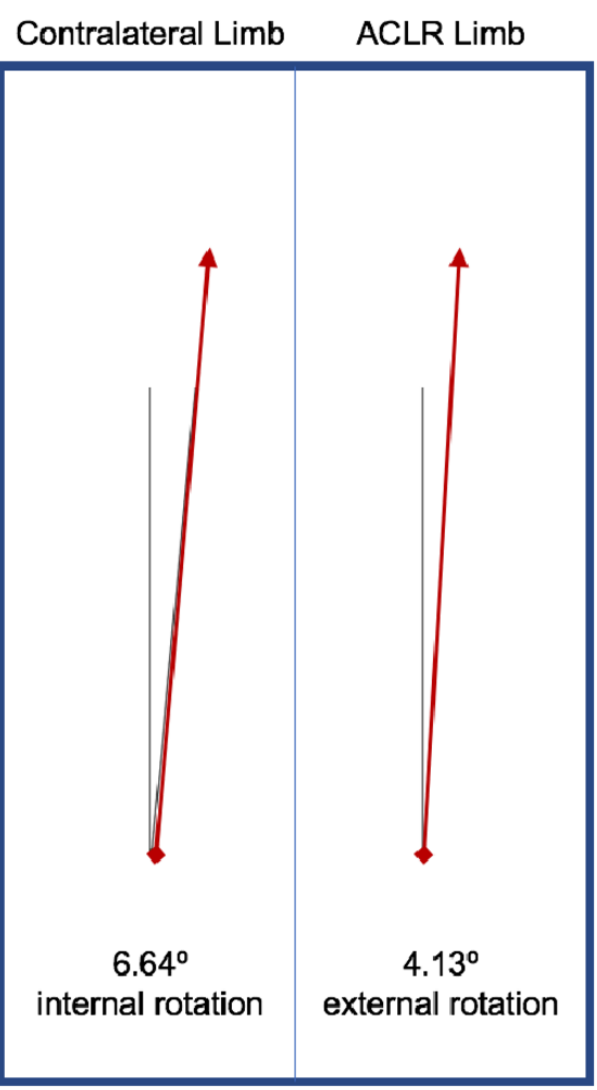

Figure 2 Prefatigue bird's eye representation of the treadmill. The right half represents transverse plane foot kinematics of ACLR limbs, the left half represents contralateral limbs. The grey line symbolises the line of gait progression and the red arrow is the mean foot progression angle (foot rotation). The mean difference in rotation of $10.78^{\circ}$ was statistically significant. ACLR, anterior cruciate ligament reconstruction.

ensure that they exerted themselves fully. The leg press was performed first with the contralateral limb, then the ACLR limb. Single leg power tests were performed to determine the weight used during the fatigue protocol. For each limb, the leg press was set to $50 \%$ of the weight at which maximum power was achieved.

\section{Data and statistical analysis}

Based on previous studies investigating the effect of fatigue on lower limb biomechanics, we estimated that a sample size of 15 would be required to achieve $80 \%$ statistical power and an alpha level of $0.05 .{ }^{1325}$

Data were collected via force plates embedded within the treadmill and the average FPA for each limb was calculated during a $15 \mathrm{~s}$ period. Our main outcome measure was transverse plane foot kinematics (FPA), defined as the angle between the long axis of the foot (from the heel to the second metatarsal) and the line of progression of gait (longitudinal axis of the treadmill) (figures 2 and 3). Positive values represent foot external rotation and negative values represent internal rotation relative to the line of gait progression. ACLR and contralateral limb mean FPA values were compared in the prefatigue and 


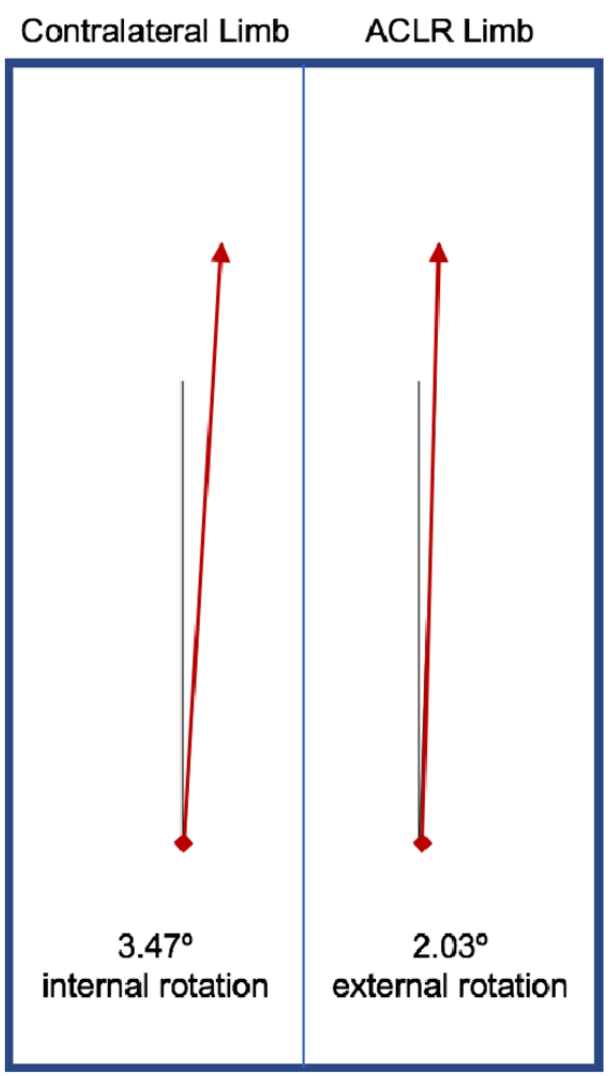

Figure 3 Postfatigue birds eye representation of the treadmill. The right half represents transverse plane foot kinematics of ACLR limb, the left half represents contralateral limbs. the grey line symbolises the line of gait progression and the red arrow is the mean foot progression angle (foot rotation). The mean difference in rotation of $5.50^{\circ}$ was not statistically significant. ACLR, anterior cruciate ligament reconstruction.

postfatigue condition. The FPA change from prefatigue to postfatigue was also compared for ACLR and contralateral limbs. A paired t-test was used for each comparison. The same three comparisons were made for dominant versus non-dominant limbs. Binary correlation was used to assess for correlation between potential confounding variables and the outcome variables. These included age, IKDC total, Lysholm score and time since surgery. An alpha level of 0.05 was used to define statistical significance. Analyses were performed using SPSS statistical software (V.22.0, SPSS).

\section{RESULTS}

All data were normally distributed with equal variance. Demographic data are presented in table 2. The mean age of subjects was 37.7 (SD 10.0). The mean postoperative time was 45.3 months (SD 15.4). The mean Tegner

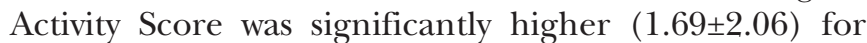
subjects prior to injury $(p=0.012)$. The $0.2 \mathrm{~km} /$ hour difference between prefatigue and postfatigue running speeds was not statistically significant $(\mathrm{p}=0.381)$.

\section{Prefatigue running biomechanics}

Prefatigue, subjects ran with externally rotated operated limb feet $\left(4.13^{\circ} \pm 10.04^{\circ}\right)$ but internally rotated contralateral feet $\left(-6.65^{\circ} \pm 8.20^{\circ}\right)$ (figure 2 ). The mean difference of $10.78^{\circ} \pm 15.12^{\circ}$ was statistically significant $(p=0.008)$ (table 3).

No significant difference was observed between the FPA of dominant and non-dominant feet $(p=0.464)$. No correlation was observed between age, IKDC total or Lysholm score and the progression angle of either foot. Time since surgery did not correlate with the FPA of operated limb feet, but a significant correlation was observed for contralateral feet; for every additional month since surgery, contralateral feet were $0.585^{\circ}$ more internally rotated $(\mathrm{p}=0.046)$ (online supplementary appendix 1).

\section{Prefatigue to postfatigue change in running biomechanics}

Mean operated limb foot external rotation reduced by $2.10^{\circ} \pm 12.77^{\circ}$ with fatigue, whereas contralateral limb feet became $3.17^{\circ} \pm 8.95^{\circ}$ more externally rotated. The mean difference in angle change from prefatigue to post-fatigue between the two groups was $5.28^{\circ} \pm 20.66^{\circ}$, but this did not reach statistical significance $(p=0.293)$ (table 3). A difference between FPA change for dominant and non-dominant limbs from the non-fatigued to the fatigued state of $6.76^{\circ}$ was not significant $(p=0.079)$.

\section{Postfatigue running biomechanics}

When fatigued, subjects ran with externally rotated operated limb feet $\left(2.03^{\circ} \pm 13.59^{\circ}\right)$ and internally rotated contralateral foot $\left(-3.47^{\circ} \pm 13.57^{\circ}\right)$. The mean FPA difference of $5.50^{\circ} \pm 26.02^{\circ}$ was not statistically significant $(\mathrm{p}=0.382)($ table 3$)$.

Dominant feet were internally rotated $\left(-6.68^{\circ} \pm 12.68\right)$ whereas non-dominant feet were externally rotated $\left(5.24^{\circ} \pm 12.20^{\circ}\right)$. The mean difference of $11.92^{\circ} \pm 17.45^{\circ}$

Table 3 The effect of fatigue on FPA for ACLR operated versus contralateral limbs

\begin{tabular}{llll}
\hline & Prefatigue & Postfatigue & \multicolumn{1}{c}{$\begin{array}{l}\text { Prefatigue to postfatigue } \\
\text { change }\end{array}$} \\
\hline ACLR limb & $4.13^{\circ}\left(10.04^{\circ}\right)$ & $2.03^{\circ}\left(13.59^{\circ}\right)$ & $-2.10^{\circ}\left(12.77^{\circ}\right)$ \\
Contralateral limb & $-6.64^{\circ}\left(8.20^{\circ}\right)$ & $-3.47^{\circ}\left(13.57^{\circ}\right)$ & $3.17^{\circ}\left(8.94^{\circ}\right)$ \\
Mean difference & $10.78^{\circ}\left(15.12^{\circ}\right)$ & $5.50^{\circ}\left(26.66^{\circ}\right)$ & $-5.28^{\circ}\left(20.66^{\circ}\right)$ \\
P value for mean difference & 0.008 & 0.382 & 0.293
\end{tabular}

Mean (SD) degrees of rotation. Positive values represent external rotation, negative values represent internal rotation.

ACLR, anterior cruciate ligament reconstruction; FPA, foot progression angle. 
was statistically significant $(p=0.010)$. No significant correlations were observed for age, time since surgery, IKDC total or Lysholm score (online supplementary appendix 1).

\section{DISCUSSION}

ACL injury as a strong predictor of subsequent injury and specific biomechanical risk factors for reinjury have been identified. To prevent reinjury and osteoarthritis post-ACLR, we need to elucidate whether sports situational factors induce high-risk lower limb biomechanics. Fatigue, synonymous with competitive sports, is frequently cited as one such factor; most ACL injuries occur towards the end of competitive matches when fatigue levels are highest. ${ }^{21-26}$ Borotikar et al demonstrated that fatigue increases hip flexion and internal rotation in addition to knee abduction and internal rotation in healthy subjects. These biomechanical alterations increase ACL injury risk. ${ }^{827-29}$ Evidence suggests that fatigue induces unique biomechanical alterations after ACL rupture; athletes develop a hip-dominant movement strategy that diminishes with fatigue. A hip dominant gait pattern could reduce reinjury risk by reducing knee joint moments, thus exposing ACL grafts to smaller loads. Such protection would be lost with fatigue. Our findings support the assertion that following ACLR athletes develop a unique, asymmetrical gait. Subjects ran with externally rotated operated limb feet but internally rotated contralateral feet. However, when fatigued, this asymmetry diminished and was no longer significant.

Foot external rotation increases knee valgus angulation and moments, increasing ACL injury risk. ${ }^{27031}$ Hewett et al showed that landing with valgus knee moments increased the risk of ACL rupture by $250 \%{ }^{32}$ This suggests that changes in FPA consequent to ACL injury actually increase reinjury risk prior to the onset of fatigue. Valgus moments would reduce loading of the medial compartment of the knee, delaying osteoarthritis progression. ${ }^{33}$ Research shows that patients with knee osteoarthritis externally rotated their foot during the stance phase of gait as a strategy to reduce arthritic pain and slow arthritis progression. ${ }^{34}$ Therefore, the gait alteration demonstrated may delay the progression of knee osteoarthritis following ACL injury but increase the risk of ACL graft rupture.

We propose that following ACLR athletes develop a knee protective movement strategy that delays osteoarthritis progression. This may, however, increase rates of ACL graft rupture. When this movement strategy is lost with fatigue, damage to the medial compartment of the knee may accelerate. Studies suggest that increased activation of the lateral hamstring, gastrocnemeus and soleus muscles is required to maintain an externally rotated foot.$^{35}$ Indeed, increased activation of the lateral hamstrings relative to the medial hamstrings has been demonstrated in subjects with knee osteoarthritis with external rotated feet. ${ }^{36}$ The increased demands placed on these muscles may render them susceptible to fatigue explaining the breakdown of this protective movement strategy. ${ }^{37-39}$

\section{Limitations}

This study has several limitations. The gait alteration demonstrated may be gender specific, with only four female subjects, we cannot confirm this. To ensure an appropriate sample size, patients were included despite differences in age, postoperative time and concurrent injuries. This has several implications; first, postoperative time had a significant interaction with prefatigue contralateral limb FPA. Second, the inclusion of concurrent injuries may alter results. For example, meniscal injuries accelerate osteoarthritis progression. Therefore, meniscal injuries (rather than the ACL injury) might explain the toe out gait alteration. Third, our subjects had medial hamstring allografts. Residual medial hamstring weakness may have produced a relative increase in lateral hamstring activity, thus increasing operated limb external foot rotation. Using contralateral limbs as controls minimised confounding from variables such as age.

Study subjects were at least 1-year post-ACLR, providing sufficient time to complete rehabilitation. Subjects were on average 45 months post-ACLR (table 2). The rationale for not excluding subjects over a certain postoperative time was that functional deficits will remain unaddressed and persist regardless of how much time had passed.

This study adopted a closed chain exercise designed to induce fatigue in all lower limb muscle groups, inducing peripheral muscular fatigue but not accounting for the central effects of fatigue. However, it allowed utilisation of an objective measure of fatigue; reduction in lower limb power output of over $30 \%$. The subjectivity of perceived fatigue would add a source of confounding. Furthermore, it is reassuring that other studies have demonstrated that neither the level of fatigue nor the precise nature of fatigue protocols alter lower limb biomechanical findings. ${ }^{40}$ However, we cannot be certain that fatiguing ACLR limbs second did not impact on findings. Subjects ran barefoot which does not simulate competitive sport but was felt to be preferable to confounding from differences in footwear. Furthermore, running on a hard treadmill does not precisely simulate running on grass.

Regarding the risk of bias, tester blinding was not possible for either data collection or analysis. Participants could not be blinded but were unaware of the specific outcome measures being tested.

\section{CONCLUSIONS}

Functional testing after ACLR provides information relevant to the sporting context that cannot be obtained by simpler clinical tests. We have provided further evidence of alterations in functional performance after ACLR in recreational athletes following RTP. The implications of these findings to both the risk of reinjury and long-term sequelae need to be established. We propose that this gait alteration could delay osteoarthritis progression with a consequent increased 
risk of ACL graft rupture. ${ }^{33}$ It may, however, have the opposite effect on the contralateral knee. The fatigue response (manifested as reduced operated limb foot external rotation) may represent a breakdown of this knee protective movement strategy. ${ }^{41}$ A multicentre, prospective study could determine how fatigue influences reinjury risk to both operated and contralateral knees. The relevance of these findings to longer term complications such as osteoarthritis will be harder to determine. If associations are established, they could be used to improve rehabilitation strategies and RTP functional tests, therefore, enhancing our ability to safely return athletes to their preinjury competitive level.

\section{Contributors Main author of the submitted manuscript.}

Funding The authors have not declared a specific grant for this research from any funding agency in the public, commercial or not-for-profit sectors.

Competing interests None declared.

Patient consent for publication Not required.

Ethics approval Ethical approval was obtained from the UCL research ethics committee (REC) and the NHS research and development office (REC reference: 13/ YH/0343, project ID: 91530).

Provenance and peer review Not commissioned; externally peer reviewed.

Open access This is an open access article distributed in accordance with the Creative Commons Attribution Non Commercial (CC BY-NC 4.0) license, which permits others to distribute, remix, adapt, build upon this work non-commercially, and license their derivative works on different terms, provided the original work is properly cited, appropriate credit is given, any changes made indicated, and the use is non-commercial. See: http://creativecommons.org/licenses/by-nc/4.0/.

\section{REFERENCES}

1. Paterno MV, Schmitt LC, Ford KR, et al. Biomechanical measures during landing and postural stability predict second anterior cruciate ligament injury after anterior cruciate ligament reconstruction and return to sport. Am J Sports Med 2010;38:1968-78.

2. Pinczewski LA, Lyman J, Salmon LJ, et al. A 10-year comparison of anterior cruciate ligament reconstructions with hamstring tendon and patellar tendon autograft: a controlled, prospective trial. Am J Sports Med 2007;35:564-74.

3. Waldén $M$, Hägglund $M$, Ekstrand J. High risk of new knee injury in elite footballers with previous anterior cruciate ligament injury * commentary. Br J Sports Med 2006;40:158-62.

4. Thomeé R, Kaplan Y, Kvist J, et al. Muscle strength and hop performance criteria prior to return to sports after ACL reconstruction. Knee Surg Sports Traumatol Arthrosc 2011;19:1798-805.

5. Ericsson YB, Roos EM, Frobell RB. Lower extremity performance following $A C L$ rehabilitation in the KANON-trial: impact of reconstruction and predictive value at 2 and 5 years. $\mathrm{Br} J$ Sports Med 2013;47:980-5.

6. Salmon L, Russell V, Musgrove T, et al. Incidence and risk factors for graft rupture and contralateral rupture after anterior cruciate ligament reconstruction. Arthroscopy: The Journal of Arthroscopic \& Related Surgery 2005;21:948-57.

7. Shelbourne KD, Gray T, Haro M. Incidence of subsequent injury to either knee within 5 years after anterior cruciate ligament reconstruction with patellar tendon autograft. Am J Sports Med 2009;37:246-51.

8. Borotikar BS, Newcomer R, Koppes R, et al. Combined effects of fatigue and decision making on female lower limb landing postures: central and peripheral contributions to ACL injury risk. Clin Biomech 2008;23:81-92.

9. Rampinini E, Impellizzeri FM, Castagna C, et al. Effect of matchrelated fatigue on short-passing ability in young soccer players. Med Sci Sports Exerc 2008;40:934-42.

10. Hantes ME, Tsarouhas A, Giakas G, et al. Effect of fatigue on tibial rotation after single- and Double-Bundle anterior cruciate ligament reconstruction a 3-dimensional kinematic and kinetic
Matched-Group analysis. American Journal of Sports Medicine 2012:40:2045-51.

11. Kuenze C, Hertel J, Weltman A, et al. Jogging biomechanics after exercise in individuals with ACL-reconstructed knees. Med Sci Sports Exerc 2014;46:1067-76.

12. Thomas AC, Lepley LK, Wojtys EM, et al. Effects of neuromuscular fatigue on quadriceps strength and activation and knee biomechanics in individuals Post-Anterior cruciate ligament reconstruction and healthy adults. J Orthop Sports Phys Ther 2015:45:1042-50.

13. Webster KE, Santamaria LJ, McClelland JA, et al. Effect of fatigue on landing biomechanics after anterior cruciate ligament reconstruction surgery. Med Sci Sports Exerc 2012;44:910-6.

14. Webster KE, Austin DC, Feller JA, et al. Symmetry of squatting and the effect of fatigue following anterior cruciate ligament reconstruction. Knee Surg Sports Traumatol Arthrosc 2015;23:3208-13.

15. Bennour S, Ulrich B, Legrand $\mathrm{T}$, et al. Effects of foot progression angle on knee biomechanics during gait modification. Comput Methods Biomech Biomed Engin 2017;20:17-18.

16. Favre J, Jolles BM. Gait analysis of patients with knee osteoarthritis highlights a pathological mechanical pathway and provides a basis for therapeutic interventions. EFORT Open Rev 2016;1:368-74.

17. Binkley JM, Stratford PW, Lott SA, et al. The lower extremity functional scale (LEFS): Scale development, measurement properties, and clinical application. Physical Therapy 1999;79:371-83.

18. Briggs KK, Lysholm J, Tegner Y, et al. The reliability, validity, and responsiveness of the Lysholm score and Tegner activity scale for anterior cruciate ligament injuries of the knee: 25 years later. $A m \mathrm{~J}$ Sports Med 2009;37:890-7.

19. Irrgang JJ, Anderson AF, Boland AL, et al. Development and validation of the International knee documentation Committee subjective knee form. Am J Sports Med 2001;29:600-13.

20. Hanke TA, Rogers MW. Reliability of ground reaction force measurements during dynamic transitions from bipedal to singlelimb stance in healthy adults. Phys Ther 1992;72:810-6.

21. Kram R, Griffin TM, Donelan JM, et al. Force treadmill for measuring vertical and horizontal ground reaction forces. J Appl Physiol 1998;85:764-9.

22. Cortes N, Quammen D, Lucci S, et al. A functional agility short-term fatigue protocol changes lower extremity mechanics. J Sports Sci 2012;30:797-805.

23. Lucci S, Cortes N, Van Lunen B, et al. Knee and hip sagittal and transverse plane changes after two fatigue protocols. J Sci Med Sport 2011;14:453-9.

24. Miura K, Ishibashi Y, Tsuda E, et al. The effect of local and general fatigue on knee proprioception. Arthroscopy: The Journal of Arthroscopic \& Related Surgery 2004;20:414-8.

25. Ferber R, Davis IM, Williams DS. Gender differences in lower extremity mechanics during running. Clin Biomech 2003;18:350-7.

26. Hawkins RD, Hulse MA, Wilkinson C, et al. The association football medical research programme: an audit of injuries in professional football. Br J Sports Med 2001;35:43-7.

27. Chappell JD, Yu B, Kirkendall DT, et al. A comparison of knee kinetics between male and female recreational athletes in stop-jump tasks. Am J Sports Med 2002;30:261-7.

28. Benjaminse A, Habu A, Sell TC, et al. Fatigue alters lower extremity kinematics during a single-leg stop-jump task. Knee Surg Sports Traumatol Arthr 2008;16:400-7.

29. Chappell JD, Herman DC, Knight BS, et al. Effect of fatigue on knee kinetics and kinematics in stop-jump tasks. Am J Sports Med 2005;33:1022-9.

30. McLean SG, Huang X, van den Bogert AJ. Association between lower extremity posture at contact and peak knee valgus moment during sidestepping: implications for ACL injury. Clin Biomech 2005;20:863-70

31. Sigward SM, Powers CM. Loading characteristics of females exhibiting excessive valgus moments during cutting. Clin Biomech 2007;22:827-33.

32. Hewett TE, Myer GD, Ford KR, et al. Biomechanical measures of neuromuscular control and valgus loading of the knee predict anterior cruciate ligament injury risk in female athletes: a prospective study. Am J Sports Med 2005;33:492-501.

33. Miyazaki T, Wada M, Kawahara $\mathrm{H}$, et al. Dynamic load at baseline can predict radiographic disease progression in medial compartment knee osteoarthritis. Ann Rheum Dis 2002;61:617-22.

34. Chang A, Hurwitz D, Dunlop D, et al. The relationship between toe-out angle during gait and progression of medial tibiofemoral osteoarthritis. Ann Rheum Dis 2007:66:1271-5. 
35. Lynn SK, Kajaks T, Costigan PA. The effect of internal and external foot rotation on the adduction moment and lateral-medial shear force at the knee during gait. J Sci Med Sport 2008;11:444-51.

36. Lynn SK, Costigan PA. Effect of foot rotation on knee kinetics and hamstring activation in older adults with and without signs of knee osteoarthritis. Clin Biomech 2008;23:779-86.

37. Manabe Y, Shimada K, Ogata M. Effect of slow movement and stretch-shortening cycle on lower extremity muscle activity and joint moments during squat. J Sports Med Phys Fitness 2007;47:1-12.

38. Nyland JA, Caborn DNM, Shapiro R, et al. Fatigue after eccentric quadriceps femoris work produces earlier gastrocnemius and delayed quadriceps femoris activation during crossover cutting among normal athletic women. Knee Surgery, Sports Traumatology, Arthroscopy 1997;5:162-7.

39. Padua DA, Arnold BL, Perrin DH, et al. Fatigue, vertical leg stiffness, and stiffness control strategies in males and females. J Athl Train 2006;41:294-304.

40. McLean SG, Fellin RE, Felin RE, et al. Impact of fatigue on gender-based high-risk landing strategies. Med Sci Sports Exerc 2007;39:502-14.

41. Thomeé R, Neeter C, Gustavsson A, et al. Variability in leg muscle power and hop performance after anterior cruciate ligament reconstruction. Knee Surg Sports Traumatol Arthrosc 2012;20:1143-51. 